Proceedings

\title{
Enhancing the Sensitivity of SMS Fiber Sensors by the Use of High Refractive Index Coatings ${ }^{\dagger}$
}

\author{
Thulio Vette 1, Gustavo Burns ${ }^{1}$, Renato Bellini ${ }^{1}$, Sergio S. Camargo Jr. ${ }^{2}$, Carolina V. S. Borges ${ }^{1}$, \\ Bruno Rente ${ }^{1,3, *}$, Kenneth Grattan ${ }^{3}$ and Maria A. G. Martinez ${ }^{1}$ \\ 1 Electronic Engineering Department, Centro Federal de Educação Tecnológica Celso Suckow da \\ Fonseca-CEFET/RJ, Rio de Janeiro 20271-110, Brazil; thulio.vette@aluno.cefet-rj.br (T.V.); \\ gustavo.soares@aluno.cefet-rj.br (G.B.); renato.bellini@aluno.cefet-rj.br (R.B.); \\ carolina.borges@cefet-rj.br (C.V.S.B.); maria.martinez@cefet-rj.br (M.A.G.M.) \\ 2 Metalurgy and Materials Engineering Program-Coppe, Universidade Federal do Rio de Janeiro, \\ Rio de Janeiro 21949-900, Brazil; camargo@metalmat.ufrj.br \\ 3 School Mathematics, Computer Science and Engineering, City University of London, London EC1V 0HB, UK; \\ k.t.v.grattan@city.ac.uk \\ * Correspondence: bruno.rente@city.ac.uk (B.R.); Tel.: +44-7407684247 \\ + Presented at the Eurosensors 2018 Conference, Graz, Austria, 9-12 September 2018. \\ Published: 7 December 2018
}

\begin{abstract}
This paper presents a study on the behavior of single-mode/multimode/single-mode sensors with diamond-like carbon coating of high refractive index. Spectra and response for different values of the external medium refractive index are recorded and analyzed.
\end{abstract}

Keywords: fiber optic sensors; Interferometry; Subwavelength structures

\section{Introduction}

Single-mode/Multimode/Single-mode sensors (SMS) are interferometric devices that use multimode interference (MMI) as the working principle for temperature, bending and refractive index measurements. Since the discovery of the self-imaging phenomenon [1], SMS sensors have been widely applied for their high sensitivity to refractive index and their straightforward, low-cost manufacturing method [2].

SMS sensors characteristics are comparable to those more traditionally used for refractive index measurements. Methods such as long-period gratings (LPG) [3] and surface Plasmon resonance (SPR) [4] also have excellent results but with far more costly and complicated manufacturing.

A common drawback for all the above-mentioned technologies is their asymptotic behavior for specific ranges of external refractive index. The evanescent wave penetrates further into the external medium thus a maximum sensitivity is achieved only for this small interval of values which are the ones closest from the cladding [5]. This directly affects the range of applications in which the sensor can be employed.

The use of a high refractive index thin film affects the sensitivity of the sensor by shaping and broadening the field within the waveguide as well as the evanescent field in the boundary film-external medium. This technique was proved to be useful for tuning the region of maximum sensitivity in many applications and in combination with some of the methods mentioned earlier, i.e., LPG [6] and SPR [7] sensors, and others such as filters [8].

In this paper we discuss its use of diamond-like carbon (DLC) as a high refractive index (RI) coating for SMS sensors. DLC optical characteristics can be controlled by the deposition parameters, making it possible to create films with different values of RI [9]. 


\section{Materials and Methods}

The first step in creating the sensor is to create the SMS structure in which the mode interference will occur. A no-core fiber is spliced between two single-mode pigtails. This scheme guarantees the sensitivity of the device to changes in the external medium refractive index. The multi-mode section of the device is then coated with the high refractive index layer of diamond-like carbon.

The film deposition was performed using the radio-frequency plasma enhanced chemical vapor deposition (RF-PECVD) at $13.56 \mathrm{MHz}$. The fibers were cleaned with isopropanol prior to the deposition and placed in the chamber parallel to the cathode. Both sides of the fibers were exposed, by turning them 180 degrees after the first deposition and repeating the process to ensure film uniformity while still assuring that the deposited amorphous carbon takes the DLC characteristics.

Methane gas $\left(\mathrm{CH}_{4}\right)$ was used as the precursor gas at a flow of $21.5 \mathrm{sccm}$ and pressure of $1.5 \mathrm{~Pa}$ in the chamber. The deposition cathode was maintained at self-biases of $-100 \mathrm{~V},-150 \mathrm{~V}$ and $-200 \mathrm{~V}$ in order to vary the film RI. Deposition durations were then calculated to achieve a film thickness of $100 \mathrm{~nm}$ (60 min, $40 \mathrm{~min}$ and $30 \mathrm{~min}$, respectively).

The refractive index measurements were taken with the SMS sensor within a glass container to expose the structure surface to several aqueous solutions of ethylene glycol, as shown in Figure 1. Using different ethylene glycol concentrations the refractive index of the solutions changes from 1.33 (pure water) to the fiber refractive index (1.44). The container also reduces undesirable movement of the fiber.

Figure 1 shows the setup for refractive index measurements. An ASE was used as light source (S5FC1005S) and the transmitted spectra were acquired by an optical spectrum analyzer (JDSU, MTS 6000).
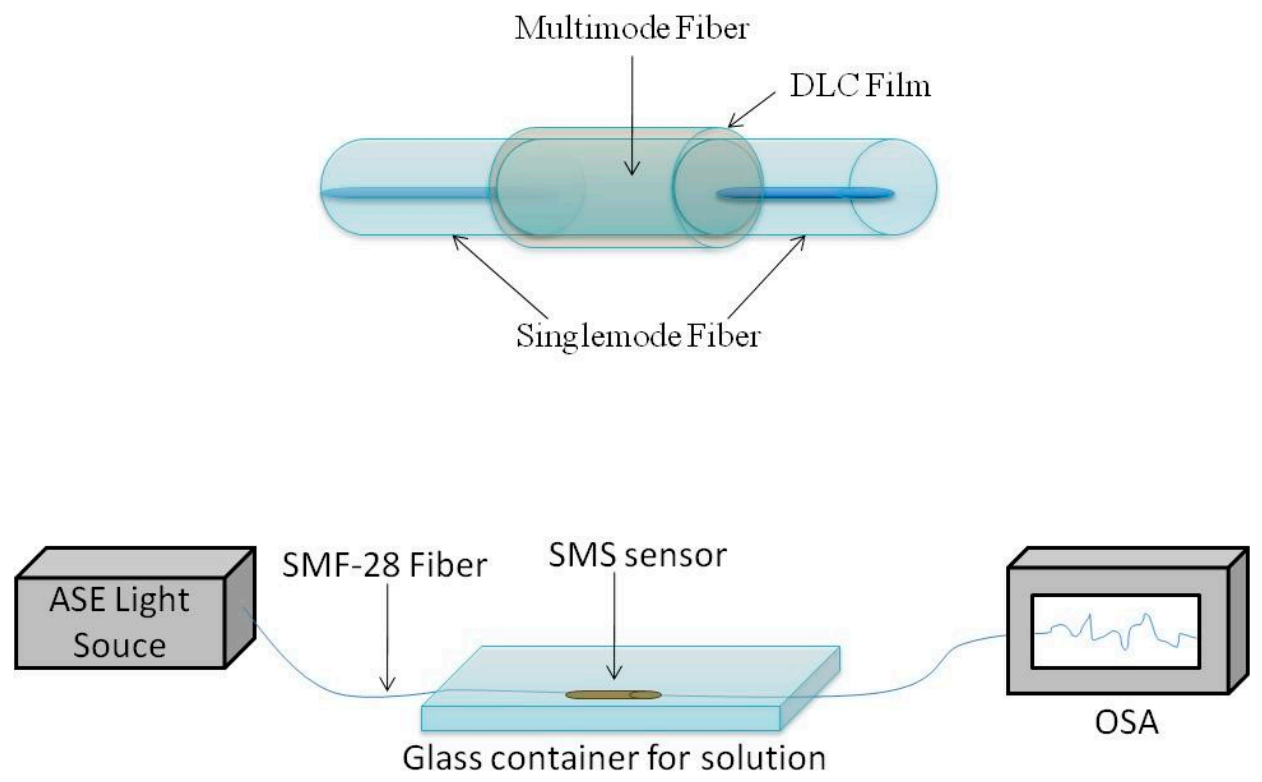

Figure 1. Schematics of a coated SMS sensor and experimental setup for refractive index measurement.

\section{Results and Discussion}

Sensors were measured in the conditions described above and their spectra were plotted. Figure 2 shows typical responses of SMS sensors for the purpose of comparison. Figure 2a corresponds to the spectra of the uncoated sensor and Figure $2 \mathrm{~b}$ corresponds to the sensor deposited with $-150 \mathrm{~V}$ of self-bias. The red curve corresponds to a higher external medium refractive index than the black curve, illustrating how the spectra drift according to the refractive index measurement. 


\section{a}

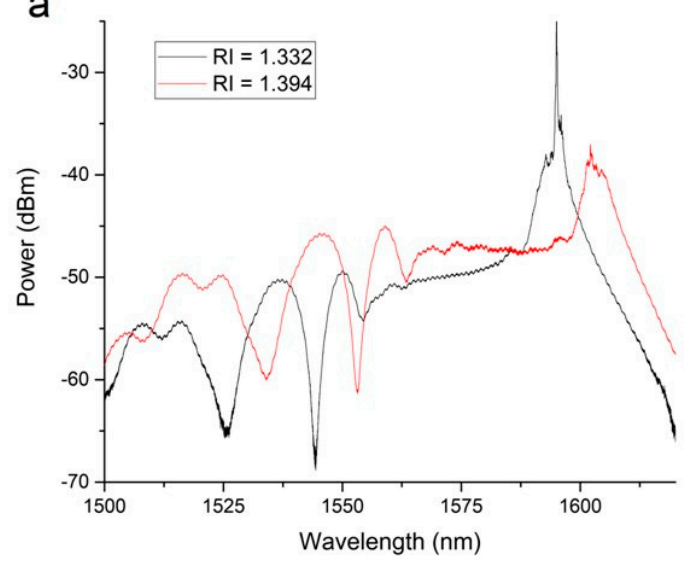

b

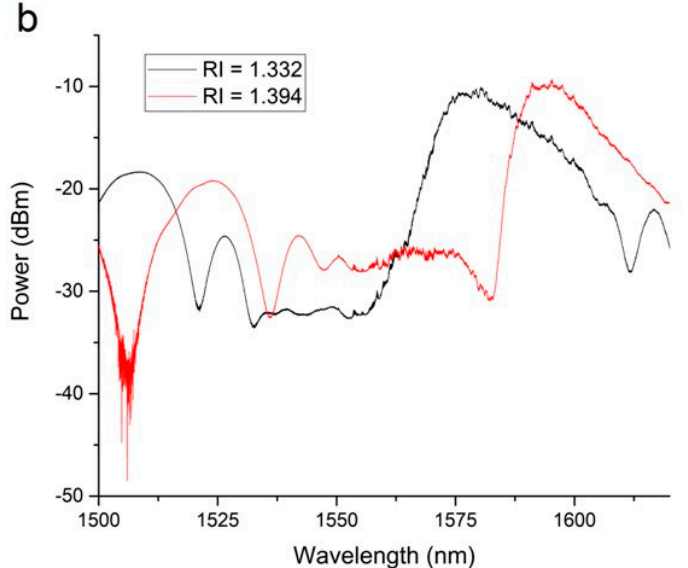

Figure 2. Spectra of SMS sensors exposed to external media with different values of refractive indexes. Results show uncoated sensors (a) and sensors coated with $-150 \mathrm{~V}$ self-bias diamond-like carbon film (b).

Comparing the graphs from different sensors, a very disparate spectrum was obtained, which indicates the interference pattern changed completely. It will also be seen when comparing sensitivities and transmission power. The wavelength shift of peaks and valleys were taken and associated with the external media refractive index. The integral of the whole spectrum was used for quantification of the transmitted power of the devices.

Figure 3a shows the wavelength shift of those peaks and valleys as a function of the external medium refractive index. For the coated fibers deposited with $-100 \mathrm{~V}$ and $-200 \mathrm{~V}$ had their sensitivities for the lower refractive indexes slightly increased as we can see in the value of their slope for indexes lower than 1.38 RIU. On the other hand, the fiber coated with a self-bias of $-150 \mathrm{~V}$ exhibited a different behavior, having its maximum sensitivity measured around 1.39 RIU.

a

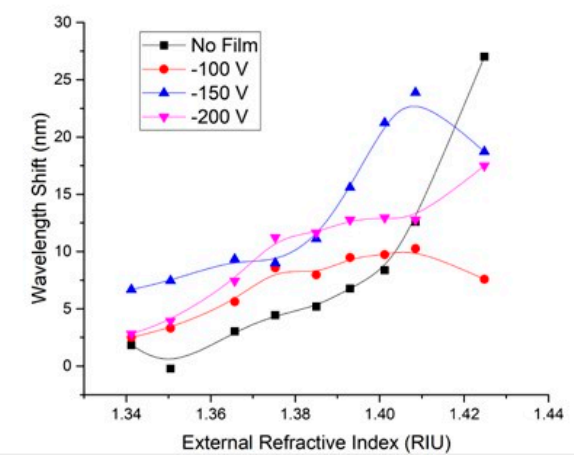

$\mathrm{b}$

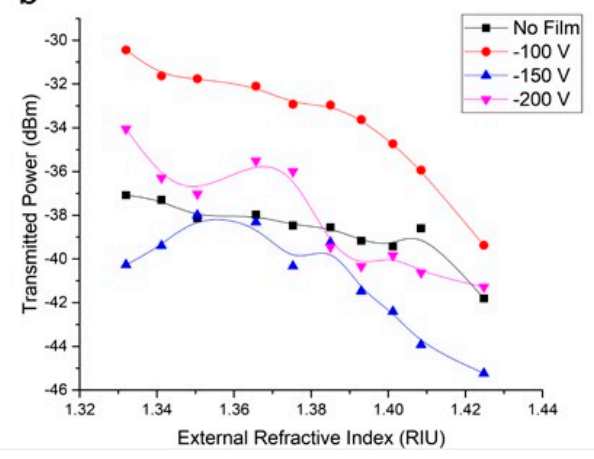

Figure 3. Wavelength shift (a) and transmitted power (b) for the different self-bias on coated sensors and the comparison of the uncoated sensor.

Additionally, the losses for the $-200 \mathrm{~V}$ are naturally higher than the ones with lower self-biases for it has a higher refractive index thus a higher effect in the effective refractive index of the radiative modes. This behavior could arise from a resonant coupling of the modes (the ones which mostly contributes to the interference) to the film as ratified by Figure $3 \mathrm{~b}$.

In summary, high refractive index films can tune the sensitivity to a more suitable span for a specific application by changing the mode field distribution, some films characteristics could accomplish this by undergoing resonances, amplifying the effect of enhancing the sensitivity.

\section{Conclusions}

Data suggests the increase of sensitivity for high refractive index coatings. It also shows a resonant behavior for specific characteristics of the film, which can be used for tuning the sensitivity 
to the desired work span. The behavior studied in this work may have implications in the applicability of SMS sensors. Such easy manufactured sensor can be modified to fit a desired application in terms of wavelength and refractive index range.

Author Contributions: B.R., C.V.S.B. and M.A.G.M. conceived and designed the experiments; T.V., G.B., S.S.C.J. and B.R., performed the experiments; T.V., G.B. and R.B. analyzed the data; S.S.C.J. contributed reagents/materials/analysis tools; B.R. and K.G. wrote the paper.

Acknowledgments: The authors would like to thank the PIBIC (Programa Institucional de Bolsas de Iniciação Científica) program from CEFET/RJ, FAPERJ (Fundação de Apoio à Pesquisa do Estado de Rio de Janeiro) and the Worshipful Company of Tin Plate Workers alias Wire Workers of the City of London for the support to this work.

Conflicts of Interest: The authors declare no conflict of interest.

\section{References}

1. Soldano, L.B.; Pennings, E.C.M. Optical multi-mode interference devices based on self-imaging: Principles and applications. J. Light. Technol. 1995, 13, 615-627, doi:10.1109/50.372474.

2. Wu, Q.; Semenova, Y.; Wang, P.; Farrell, G. High sensitivity SMS fiber structure based refractometerAnalysis and experiment. Opt. Express 2011, 19, 7937-7944, doi:10.1364/OE.19.007937.

3. James, S.W.; Tatam, R.P. Optical fibre long-period grating sensors: Characteristics and application. Meas. Sci. Technol. 2003, 14, R49-R61, doi:10.1088/0957-0233/14/5/201.

4. Homola, J.; Sinclair, S.Y.; Günter, G. Surface plasmon resonance sensors. Sens. Actuators B Chem. 1999, 54, 3-15, doi:10.1016/S0925-4005(98)00321-9.

5. Iadicicco, A.; Cusano, A.; Campopiano, S.; Cutolo, A.; Giordano, M. Thinned fiber Bragg gratings as refractive índex sensors. IEEE Sens. J. 2005, 5, 1288-1295, doi:10.1109/JSEN.2005.859288.

6. Smietana, M.; Szmidt, J.; Korwin-Pawlowski, M.L.; Bock, W.J.; Grabarczyk, J. Application of diamond-like carbon films in optical fibre sensors based on long period gratings. Diam. Relat. Mater. 2007, 16, 1374-1377, doi:10.1016/j.diamond.2006.11.018.

7. Bhatia, P.; Gupta, B.D. Surface-plasmon-resonance-based fiber-optic refractive index sensor: Sensitivity enhancement. Appl. Opt. 2011, 50, 2032-2036, doi:10.1364/AO.50.002032.

8. Constantini, D.M.; Muller, C.A.P.; Vasiliev, S.A.; Limberger, H.G.; Salathe, R.P. Tunable loss filter based on metal coated long-period fibre grating. IEEE Photonics Technol. Lett. 1999, 11, 1458-1460, doi:10.1109/68.803078.

9. Robertson, J. Diamond-like amorphous carbon. Mater. Sci. Eng. 2002, 37, 129-281, doi:10.1016/S0927-796X(02)00005-0.

(C) 2018 by the authors. Licensee MDPI, Basel, Switzerland. This article is an open access article distributed under the terms and conditions of the Creative Commons Attribution (CC BY) license (http://creativecommons.org/licenses/by/4.0/). 